\title{
Erythrodermic psoriasis de novo versus skin lesions in chronic lymphocytic leukaemia
}

\author{
Anna Słomiak-Wąsik ${ }^{1}$, Magdalena Jałowska ${ }^{1}, K^{2}$ atarzyna Iwanik ${ }^{2}$, Ryszard Żaba ${ }^{1}, Z_{\text {Zygmunt Adamski }}{ }^{1}$ \\ 1Department of Dermatology and Venereology, Poznan University of Medical Sciences, Poznan, Poland \\ ${ }^{2}$ Department of Clinical Pathology, Poznan University of Medical Sciences, Poznan, Poland
}

Adv Dermatol Allergol 2020; XXXVII (2): 277-279

DOI: https://doi.org/10.5114/ada.2020.94849

Erythroderma is an inflammatory skin disease that affects over $90 \%$ of the body surface. It is usually associated with skin desquamation and pruritus [1]. This rare and severe form affects $1-2.25 \%$ of patients diagnosed with psoriasis [2]. Differential diagnosis for erythroderma includes inter alia dandruff, atopic dermatitis, drug eruption, seborrheic dermatitis, erythrodermic stage of cutaneous lymphoma or even skin manifestation of leukaemia, including chronic lymphocytic leukaemia (CLL) $[2,3]$. Only $25 \%$ of erythroderma cases are caused by the psoriasis [4].

Skin lesions are present in 5-25\% of CLL patients [5]. The most common type is a lump or wart associated with limited lymphocyte B skin infiltration. Exfoliative dermatitis, also manifested as erythroderma have been reported in CLL [6].

Skin lesions associated with CLL might develop primarily as a skin leukaemia (manifested as blisters, ulcerations, eczema and gingival overgrowth) or secondary to hematologic or autoimmune diseases associated with CLL (e.g. skin neoplasm, petechia, exfoliative dermatitis, erythroderma or pemphigoid) [7, 8].

A 56-year-old man diagnosed with psoriasis vulgaris was referred to our department due to scaly lesions on the elbows. The patient had a 1-year history of progressively deteriorating skin lesions, but no previous medical files were available for review. No co-morbidities, oral medication intake and significant family history was reported by the patient. Previous treatment of psoriatic skin lesions included topical prescription ointment, though no information regarding ointment composition were available. Also no general symptoms such as weight loss or fever were noted. At admission erythroderma associated with itch (without desquamation) and mild ankle oedema was reported (Figure 1). Apart from that, numerous, swollen, painless lymph nodes were noted in the following locations: right lateral cervical triangle, bilateral supraclavicular area, and bilateral axillary area. The lymph node in the right lateral cervical triangle was modelling shape of the neck, what was noted by the patient 1 year ago. Due to no associated pain the patient decided not to report this finding to his general physician, also no lymph node physical examination was carried out in the preceding year.

An elevated lymphocyte count $\left(7.01 \times 10^{3} / \mu\right.$ l, cutoff level: $\left.4.50 \times 10^{3} / \mu \mathrm{l}\right)$ and white blood cell count $(12.48 \times$ $10^{3} / \mu$ l, cutoff level: $11.00 \times 10^{3} / \mu$ l) were found. Other de-

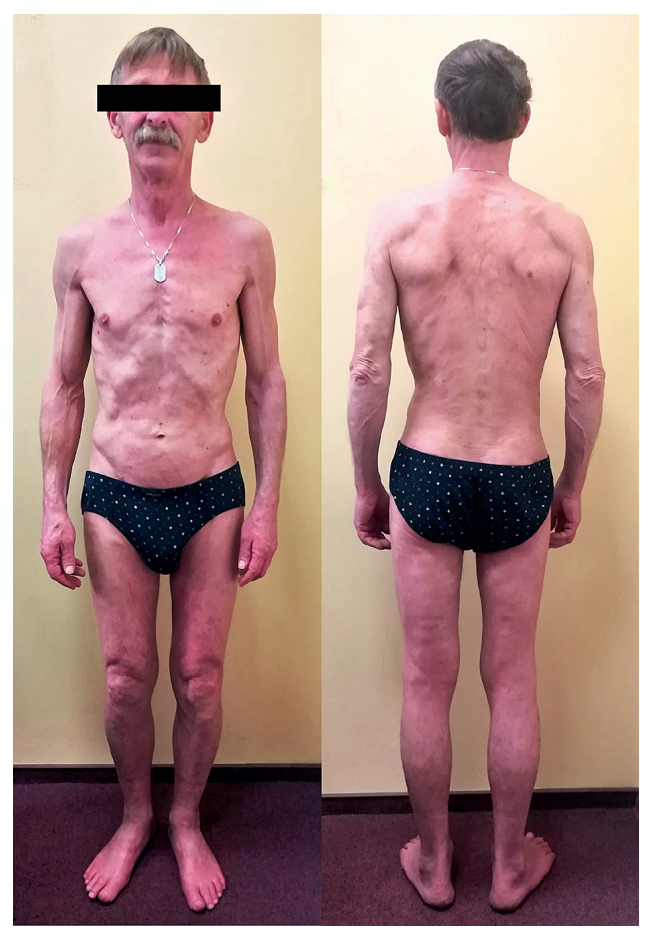

Figure 1. Dermatological status at admission - erythroderma

Address for correspondence: Anna Stomiak-Wąsik, Department of Dermatology and Venereology, Poznan University of Medical Sciences, 49 Przybyszewskiego St, 60-355 Poznan, Poland, e-mail: anna.slomiak@gmail.com Received: 13.12.2018, accepted: 11.02.2019. 


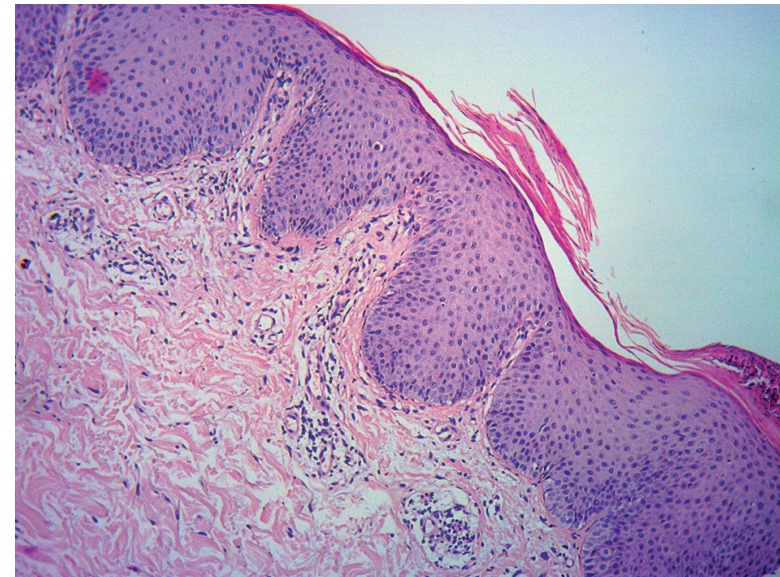

Figure 2. Histopathological examination of the skin biopsy (H\&E staining, 100× magnification)

viations included fasting hyperglycaemia and hypercholesterolemia. Both the C-reactive protein level and erythrocyte sedimentation rate were within normal ranges.

Ultrasound examination revealed multiple, heterogeneous, enlarged lymph nodes in the submandibular area, along the sternocleidomastoid muscle, supraclavicular fossa and bilaterally in the axilla. Adipose sinus was not observed in the part of the lymph nodes.

Next, the patient was examined by the haematologist. Microscopic examination of the blood sample, cytologic examination of the bone marrow as well as bone marrow cytometry were performed. Based on available laboratory test results as well as physical examination findings, CLL Rai grade I was diagnosed.

Due to unusual skin lesion morphology and unclear medical history of the patient, skin biopsy was taken early on admission. According to the reviewing pathologist, acute psoriasis was the most probable diagnosis (Figure 2). Immunohistochemical staining ruled out leukaemia associated skin infiltration of the skin sample.

Numerous cell types are involved in the pathophysiology of the psoriasis. The most significant are Th1 lymphocytes, which produce multiple proinflammatory cytokines e.g. tumor necrosis factor (TNF) $\alpha$, TNF- $\beta$, interleukin (IL) 2, IL-3, IL-22, IL-26 as well as granulocytemacrophage colony-stimulating factor (GM-CSF). In CLL a correlation was found between TNF- $\alpha$ level and white blood cell count, lymphocyte count and CD19+/CD5+ lymphocyte count. Various responses of leukemic cells to TNF- $\alpha$ stimulation were discovered by the scientists [9]. In CLL patients TNF- $\alpha$ elevation is observed in blood serum [9-11]. It is suspected that TNF- $\alpha$ is involved in CLL progression. What is more, TNF- $\alpha$ as a proinflammatory cytokine is involved in pathophysiology of both psoriasis and CLL. We suspect that common interactions of TNF- $\alpha$ contributed to CLL development in our patient. TNF inhibitors are used successfully to treat psoriasis,
5 types of the drug are available in Poland [6]. First trials of TNF inhibitors in CLL are available. Balato et al. reported a case of a 41-year-old female diagnosed with psoriasis and CLL treated initially with etanercept [12]. After change to infliximab, both PSI index improved as well as CLL progression was stopped. After 18 months of such treatment, remission of psoriasis was still observed as well as no lymphocyte elevation was noted. Infliximab treatment is one of the available therapeutic options for our patients after approval by the haematologist. Anyway, it is worth noticing that biological treatment is contraindicated in patients with a history of malignant neoplasm in the last 5 years.

Uncertain past medical history, lack of medical files, negative family history and lack of common psoriatic skin lesions at admission encouraged authors to perform skin biopsy in order to confirm diagnosis. Additional doubts regarding condition underlying erythroderma resulted from suspicion and confirmation of CLL in the patient. Authors highlight the significance of skin biopsy examination in erythroderma origin disclosure since psoriasis underlies only one fourth of cases. Thorough physical examination and basic laboratory tests remain crucial for establishing diagnosis. Management of CLL Rai stage I includes regular follow-up by the haematologist and treatment introduction when progression occurs.

\section{Conflict of interest}

The authors declare no conflict of interest.

\section{References}

1. Okoduwa C, Lambert WC, Schwartz RA, et al. Erythroderma: review of a potentially life-threatening dermatosis. Indian J Dermatol 2009; 54: 1-6.

2. Stinco G, Errichetti E. Erythrodermic psoriasis: current and future role of biologicals. BioDrugs 2015; 29: 91-101.

3. Rym BM, Mourad M, Bechir Z, et al. Erythroderma in adults: a report of 80 cases. Int J Dermatol 2005; 44: 731-35.

4. Rosenbach M, Hsu S, Korman NJ, et al. Treatment of erythrodermic psoriasis: from the medical board of the National Psoriasis Foundation. J Am Acad Dermatol 2010; 62: 655-62.

5. Robak E, Robak T. Skin lesions in chronic lymphocytic leukemia. Leuk Lymphoma 2007; 48: 855-65.

6. Dhir R. Chronic lymphocytic leukaemia manifestating as exfoliative dermatitis. Indian J Dermatol Venereol Leprol 1995; 61: 102-3.

7. Lu C, Li L, Oiao O, et al. Cutaneous manifestations in a patient with chronic lymphocytic leukemia involving the head, neck and distal extremities. Exp Ther Med 2015; 9: 877-79.

8. Agnew KL, Ruchlemer R, Catovsky D, et al. Cutaneous findings in chronic lymphocytic leukaemia. Br J Dermatol 2004; 150: 1129-35.

9. Wąsik-Szczepanek E. Czynnik martwicy guza i jego rola w przewlekłej białaczce limfocytowej (PBL) [Tumor necrosis factor and its role in chronic lymphocytic leukemia]. Acta Haematol Pol 2012; 43: 146-9.

10. Ferrajoli A, Keating MJ, Manshouri T, et al. The clinical significance of tumor necrosis factor-alpha plasma level in 
patients having chronic lymphocytic leukemia. Blood 2002; 100: 1215-9.

11. Bojarska-Junak A, Hus I, Wasik Szczepanek E, et al. Peripheral blood and bone marrow TNF and TNF receptors in early and advanced stages of B-CLL in correlation with ZAP-70 protein and CD38 antigen. Leuk Res 2008; 32: 225-33.

12. Balato A, Lembo S, Cirillo T, et al. Anti-tumor necrosis factoralpha therapy in the management of psoriasis and B-chronic lymphocytic leukemia. Case Rep Dermatol 2011; 3: 60-3. 